\title{
Effect of Calmodulin-like Gene ( $C M L)$ Overexpression on Stilbene Biosynthesis in Cell Cultures of Vitis amurensis Rupr.
}

\author{
Olga A. Aleynova ${ }^{1}{ }^{(}$, Andrey R. Suprun ${ }^{1}{ }^{(D}$, Alexey A. Ananev ${ }^{1,2}{ }^{,}$Nikolay N. Nityagovsky ${ }^{1}$, Zlata V. Ogneva $^{1}$, \\ Alexandra S. Dubrovina ${ }^{1}$ (I) and Konstantin V. Kiselev ${ }^{1, *}$ \\ 1 Laboratory of Biotechnology, Federal Scientific Center of the East Asia Terrestrial Biodiversity, FEB RAS, \\ 690022 Vladivostok, Russia; aleynova@biosoil.ru (O.A.A.); suprun.hi@gmail.com (A.R.S.); \\ lexxdance@mail.ru (A.A.A.); niknit1996@gmail.com (N.N.N.); zlata.v.ogneva@gmail.com (Z.V.O.); \\ dubrovina@biosoil.ru (A.S.D.) \\ 2 Department of Biochemistry and Biotechnology, Institute of the World Ocean, Far Eastern Federal University, \\ 690090 Vladivostok, Russia \\ * Correspondence: kiselev@biosoil.ru; Tel.: +8-423-2310410; Fax: +8-4232-310193
}

check for updates

Citation: Aleynova, O.A.; Suprun,

A.R.; Ananev, A.A.; Nityagovsky,

N.N.; Ogneva, Z.V.; Dubrovina, A.S.; Kiselev, K.V. Effect of Calmodulin-like Gene (CML) Overexpression on Stilbene Biosynthesis in Cell Cultures of Vitis amurensis Rupr. Plants 2022, 11, 171. https://doi.org/10.3390/ plants11020171

Academic Editor: Othmane Merah

Received: 28 December 2021

Accepted: 5 January 2022

Published: 10 January 2022

Publisher's Note: MDPI stays neutral with regard to jurisdictional claims in published maps and institutional affiliations.

Copyright: (c) 2022 by the authors. Licensee MDPI, Basel, Switzerland. This article is an open access article distributed under the terms and conditions of the Creative Commons Attribution (CC BY) license (https:// creativecommons.org/licenses/by/ $4.0 /)$.

\begin{abstract}
Stilbenes are plant phenolics known to rapidly accumulate in grapevine and other plants in response to injury or pathogen attack and to exhibit a great variety of healing beneficial effects. It has previously been shown that several calmodulin-like protein $(C M L)$ genes were highly up-regulated in cell cultures of wild-growing grapevine Vitis amurensis Rupr. in response to stilbene-modulating conditions, such as stress hormones, UV-C, and stilbene precursors. Both CML functions and stilbene biosynthesis regulation are still poorly understood. In this study, we investigated the effect of overexpression of five $\mathrm{VaCML}$ genes on stilbene and biomass accumulation in the transformed cell cultures of $V$. amurensis. We obtained 16 transgenic cell lines transformed with the VaCML52, VaCML65, VaCML86, VaCML93, and VaCML95 genes (3-4 independent lines per gene) under the control of the double CaMV 35S promoter. HPLC-MS analysis showed that overexpression of the $\mathrm{VaCML65}$ led to a considerable and consistent increase in the content of stilbenes of 3.8-23.7 times in all transformed lines in comparison with the control calli, while biomass accumulation was not affected. Transformation of the $V$. amurensis cells with other analyzed $V a C M L$ genes did not lead to a consistent and considerable effect on stilbene biosynthesis in the cell lines. The results indicate that the $\mathrm{VaCML65}$ gene is implicated in the signaling pathway regulating stilbene biosynthesis as a strong positive regulator and can be useful in viticulture and winemaking for obtaining grape cultivars with a high content of stilbenes and stress resistance.
\end{abstract}

Keywords: CML; calcium; stilbenes; transgenic cells; plant cell cultures

\section{Introduction}

Stilbenes are part of a vast group of plant polyphenolic compounds with antimicrobial activities synthesized via the phenylpropanoid pathway by a broad range of unrelated plant families [1-3]. Plant stilbenes are well-known to confer a variety of health benefits and possess promising pharmacological applications [4-6]. Stilbenes are also classified as phytoalexins that rapidly accumulate in plants in response to injury or microbial pathogens and contribute to plant environmental stress resistance [3,7]. Trans-resveratrol ( $t$-resveratrol) is the most prominent stilbene with a variety of valuable biologically active properties and was widely used in the food and medicine industries [8]. Stilbenes are known to be involved in plant constitutive and inducible defense reactions against plant fungal and bacterial pathogens, nematodes, and herbivores [7,9]. Biosynthesis of natural stilbenoids is activated in response to a number of other environmental stresses, such as ultraviolet (UV) irradiation, wounding, drought, or unfavorable temperatures [3,9].

Although there is a lack of information about the signaling pathways and biochemical events regulating stilbene biosynthesis in plant tissues, it is known that (1) hormone 
signaling (salicylic acid (SA), methyl jasmonate (MeJA), ethylene, jasmonic acid, and abscisic acid) [10-15]; (2) production of reactive oxygens species (ROS) [10,12,15]; (3) stressinduced increase in calcium cytoplasmic concentration $[10,11,16,17]$, and (4) activation of calcium-dependent protein kinases or CDPKs [18-20] are implicated in the activation of stilbene biosynthesis in plant tissues. These signaling events eventually lead to the activation of specific transcription factors responsible for the induction of stilbene synthases (STSs) and other stilbene biosynthetic genes [13,21,22]. Furthermore, our recent results demonstrated high responsiveness of plant calmodulin-like protein (CML) genes to the agents stimulating the biosynthesis of stilbenes, including stilbene precursors, SA and MeJa, and UV-C exposure [23], which suggested plant CML genes as promising candidates for the activation of plant defense reactions and stilbene production. Thus, it is known that calcium signaling is implicated in the regulation of stilbene biosynthesis in grapevine [3].

Plants encode several classes of calcium sensor proteins, which perceive and decode the alterations in calcium cytoplasmic concentration induced in response to stress and developmental cues $[24,25]$. The plant calcium sensor proteins include calmodulins (CaMs), calmodulin-like proteins (CMLs), calcium-dependent protein kinases (CDPKs), calcineurin B-like proteins (CBLs), and CBL-interacting protein kinases (CIPKs) [26,27]. Most of the calcium sensor protein subfamilies are encoded by multigene families where the most numerous are CMLs, including 50 genes in Arabidopsis [28] and at least $54 C M L$ genes in grapevine [29]. While the high number and diversity of plant CMLs suggest their importance in plant survival and productivity, the biological functions of this class of plant calcium-binding proteins remain poorly studied.

CML proteins were classified as "sensor relays" lacking "sensor responder" domains and catalytic activity $[26,30]$. After calcium binding, CMLs undergo conformational changes and interact with their target proteins whose activity is then altered. CMLs transmit the information from the environmental and developmental cues to various downstream biochemical events leading to a proper physiological response. The available research findings show that plant CMLs are involved in the regulation of various developmental processes, such as trichome branching or seed development, and plant abiotic and biotic stress responses [31-34]. It was shown that the expression of particular CML genes was activated in response to pathogen attack [35] or to stress hormones, such as methyl jasmonate or MeJA (AtCML39) and salicylic acid or SA (AtCML43) [36-38]. A number of studies show that overexpression of $C M L$ genes in plants improved plant abiotic stress resistance or contributed to plant-pathogen protection [39-43]. For example, CaCML13 acts positively in pepper immunity against Ralstonia solanacearum infection forming a feedback loop with CabZIP63 transcriptional factor [35].

At present, the relationship of plant CMLs and other calcium sensor proteins with plant defense reactions and production of plant secondary metabolites remains almost unexplored. Recently, it was shown that a number of CML genes, such as VaCML52, 65,93 , and 95, were highly up-regulated in the leaves and cell cultures of wild grape Vitis amurensis Rupr. in response to stilbene precursors, stress hormones (MeJA, SA) and UV-irradiation [23], which are known as potent inductors of stilbene biosynthesis. The Amur grape, Vitis amurensis Rupr., displays a high level of stress resistance and stilbene content compared to other Vitaceae species and is now commercially cultivated [3,44-46]. Vines of $V$. amurensis are used as a resistant rootstock to breed materials for novel cultivars. Elucidating the molecular mechanisms and pathways underlying the stilbene biosynthesis in $V$. amurensis is of high interest.

In the present study, we aimed to investigate and verify the function of the VaCML52, 65,93 , and 95 genes as potential positive regulators of stilbene biosynthesis in the grapevine by overexpressing the $C M L$ genes in cell cultures of $V$. amurensis. We also transformed $V$. amurensis with the $V a C M L 86$ gene, whose expression was not essentially changed in response to the stilbene-modulating conditions, with the purpose to use it as an additional control. Results of the present investigation demonstrated that the VaCML65 gene is a

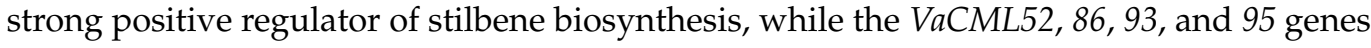


are not actively involved in this process. The VaCML65 gene can be useful in viticulture and winemaking for obtaining grape cultivars with a high content of stilbenes and resistance to environmental stresses.

\section{Results and Discussion}

\subsection{Genetic Transformation and Selection of the VaCML-Transgenic Cell Lines}

To establish Vitis amurensis Rupr. cell cultures overexpressing the full-length VaCML52, VaCML65, VaCML86, VaCML93, and VaCML95 genes and the control cell line, the V7 suspension culture of $V$. amurensis was incubated with A. tumefaciens strains to bear the pZP-RCS2$V a C M L-n p t I I$ construct for VaCML-transgenic cells or the pZP-RCS2-nptII for the control KA0 cell line. Then, we selected transgenic callus cell aggregates in the presence of 10-15 mg/L of kanamycin $(\mathrm{Km})$ for four months and established several Km-resistant lines as described [19]. The semiquantitative RT-PCR has shown that the nptII gene was transcribed in all obtained transgenic cell lines, and the absence of A.tumefaciens was confirmed using RT-PCR to control the presence or absence of the VirB2 gene (Supplementary Figure S1). The selected transformed calli represented friable vigorously growing homogenous tissues, which did not undergo differentiation on the $\mathrm{W}_{\mathrm{B} / \mathrm{A}}$ medium supplemented with 6-benzylaminopurine (BAP) and $\alpha$-naphthaleneacetic acid (NAA) in the dark. For further analysis, we used 16 transgenic cell lines transformed with the VaCML genes: three VaCML52-transformed cell lines (52-1, 52-2, 52-3), four VaCML65-transformed cell lines (65-1, 65-2, 65-3, 65-4), three VaCML86-transformed cell lines (86-1, 86-2, 86-3), three VaCML93-transformed cell lines (93-1, 93-2, 93-3), and four VaCML95-transformed cell lines (95-1, 95-2, 95-3, 95-4) (Table 1). The control KA0 transformed calli reproduced morphological, growth, and biosynthetic characteristics of the parent V7 culture.

The $\mathrm{VaCML}$-transgenic cell lines were confirmed for expression of the transgene and

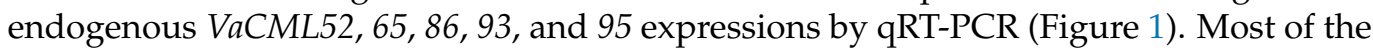
$\mathrm{VaCML}$-transformed cell lines actively expressed the transgenes (Figure 1a-e). Additionally, the total expression of $\mathrm{VaCML52}, 65,86,93$, and 95 genes exceeded that in the KA0 control in most of the VaCML-transformed calli, except for VaCML95 (Figure 1k-o). The analysis of the endogenous $\mathrm{VaCML}$ expression revealed that expression of endogenous VaCML86 and VaCML93 was not affected in all cell lines, while expression of VaCML52, 65 , and 95 was altered in a part of the cell lines obtained in comparison with that in the control KA0 (Figure 1f-j). On one side, it is known that plant transgenes and/or plant homologous endogenous genes can be silenced as a result of induction of RNA interference (RNAi) and generation of transgene-derived small interfering RNA (siRNAs) [47,48]. In the course of RNAi, double-stranded RNAs (dsRNAs) are processed by a ribonuclease into siRNAs, which are incorporated in the RNA-induced silencing complex that provides cleavage, destabilization, or hindering translation of any homologous mRNAs [49,50]. On the other side, other studies show that in contrast to a conventional transgene, an endogene-resembling transgene was more stably expressed and poorly processed into small RNAs [51,52]. Therefore, there is a need to verify whether either transgene and/or endogene was silenced and to what degree after plant genetic transformation and establishment of plant transgenic cell cultures. The data obtained in the present work show that expression of the endogenous VaCML52 and VaCML95 genes was down-regulated after transformation in the 52-2, 52-3, and 95-3 lines, which suggested that the decrease in endogenous expression, in this case, might be caused by the activation of the RNAi process. 


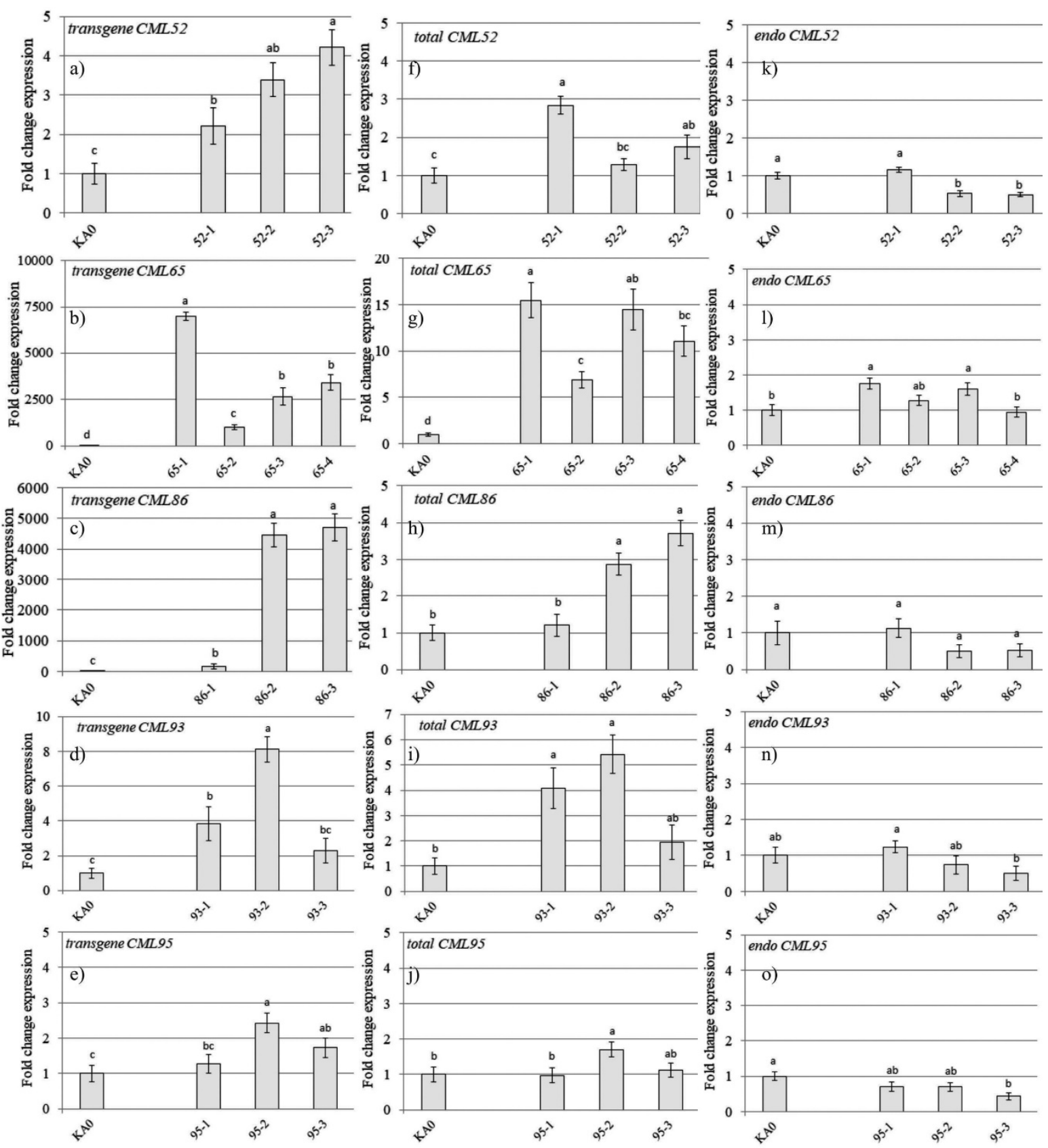

Figure 1. Quantification of the transgene $(\mathbf{a}-\mathbf{e})$, total $(\mathbf{f}-\mathbf{j})$, and endogenous $(\mathbf{k}-\mathbf{o}) \mathrm{VaCML}$ gene expression in the transgenic cells of Vitis amurensis performed by quantitative RT-PCR. RNA was extracted from control $V$. amurensis cell line transformed with the empty vector harboring only $n p t I I$ selective marker (KA0) and $V$. amurensis cell lines transformed with the VaCML52 (a,f,k, VaCML52-1, -2, and -3), VaCML65 (b,g,l, VaCML65-1, -2, -3, and -4), VaCML86 (c,h,m, VaCML86-1, -2, and -3), VaCML93 (d,i,n, VaCML93-1, -2, and -3), and VaCML95 (e,j,o, VaCML95-1, -2, -3, and -4) genes. The data are presented as mean \pm SE (two independent experiments with eight technical replicates). Means on each figure followed by the same letter were not different using one-way analysis of variance (ANOVA), followed by the Tukey HSD multiple comparison test.

\subsection{Stilbene Content and Biomass Accumulation in the Grapevine VaCML-Transgenic Cell Lines}

It is known that the highest content of stilbenes in callus cell cultures of $V$. amurensis was typical for the 35th day of cultivation [53]. Therefore, cell culture samples were collected from the 35-day-old calli for stilbene extraction and biomass analysis. In Table 1 , we presented fresh and dry biomass accumulation in the $\mathrm{VaCML}$-transformed cell lines of 
$V$. amurensis. Transformation of the $V$. amurensis cells with the analyzed $V a C M L$ genes did not substantially affect the fresh and dry growth parameters in most obtained cell lines, except for the fresh weight of the 93-3 and 95-2 cell lines (Table 1).

Table 1. Biomass accumulation and total stilbene production in the cell lines of Vitis amurensis overexpressing the VaCML52, VaCML65, VaCML86, VaCML93, or VaCML95 genes.

\begin{tabular}{|c|c|c|c|c|}
\hline Cell Line & $\begin{array}{l}\text { Overexpressed } \\
\text { CML Gene }\end{array}$ & Fresh Weight, g/L & Dry Weight, g/L & $\begin{array}{c}\text { Total Stilbene } \\
\text { Production, mg/L }\end{array}$ \\
\hline KA0 & - & $163.7 \pm 13.3^{b}$ & $6.65 \pm 1.02^{a}$ & $4.69 \pm 1.54^{\mathrm{d}}$ \\
\hline $52-1$ & \multirow{3}{*}{ VaCML52 } & $155.0 \pm 12.8^{b}$ & $8.07 \pm 1.17^{a}$ & $13.82 \pm 5.37^{c}$ \\
\hline $52-2$ & & $165.1 \pm 14.4^{b}$ & $6.77 \pm 0.82^{a}$ & $14.35 \pm 4.88^{c}$ \\
\hline $52-3$ & & $165.2 \pm 10.1^{b}$ & $7.83 \pm 0.97^{a}$ & $8.93 \pm 3.66^{\mathrm{cd}}$ \\
\hline $65-1$ & \multirow{4}{*}{ VaCML65 } & $131.3 \pm 8.5^{b}$ & $7.11 \pm 0.88^{a}$ & $135.66 \pm 34.20^{a}$ \\
\hline $65-2$ & & $195.1 \pm 15.5^{a b}$ & $6.75 \pm 0.91^{a}$ & $56.67 \pm 18.62^{b}$ \\
\hline $65-3$ & & $137.3 \pm 14.9^{b}$ & $6.91 \pm 0.93^{a}$ & $27.84 \pm 13.25^{b}$ \\
\hline $65-4$ & & $201.1 \pm 16.6^{a b}$ & $8.81 \pm 1.32^{\mathrm{a}}$ & $26.94 \pm 10.19 \mathrm{bc}$ \\
\hline $86-1$ & \multirow{3}{*}{ VaCML86 } & $158.7 \pm 11.2^{b}$ & $6.69 \pm 0.75^{a}$ & $6.42 \pm 1.88^{\mathrm{d}}$ \\
\hline $86-2$ & & $158.9 \pm 10.4^{b}$ & $6.54 \pm 0.98^{a}$ & $3.22 \pm 0.91^{d}$ \\
\hline $86-3$ & & $149.1 \pm 8.9^{b}$ & $5.71 \pm 0.74^{a}$ & $5.05 \pm 1.74^{d}$ \\
\hline $93-1$ & \multirow{3}{*}{ VaCML93 } & $197.1 \pm 13.7^{a b}$ & $8.75 \pm 1.43^{a}$ & $26.46 \pm 11.47^{b c}$ \\
\hline $93-2$ & & $153.8 \pm 12.2^{b}$ & $6.21 \pm 0.88^{a}$ & $4.31 \pm 1.12^{\mathrm{d}}$ \\
\hline $93-3$ & & $218.5 \pm 13.4^{\mathrm{a}}$ & $8.77 \pm 1.50^{\mathrm{a}}$ & $11.07 \pm 5.15^{\mathrm{c}}$ \\
\hline $95-1$ & \multirow{3}{*}{ VaCML95 } & $198.8 \pm 10.8^{a b}$ & $7.81 \pm 0.99^{a}$ & $3.78 \pm 0.74^{\mathrm{d}}$ \\
\hline $95-2$ & & $154.7 \pm 11.1^{\mathrm{b}}$ & $6.75 \pm 0.79^{a}$ & $9.28 \pm 4.13^{\mathrm{cd}}$ \\
\hline $95-3$ & & $225.2 \pm 15.6^{\mathrm{a}}$ & $8.64 \pm 1.17^{\mathrm{a}}$ & $2.81 \pm 0.65^{\mathrm{d}}$ \\
\hline
\end{tabular}

The callus tissue samples were harvested from the 35-day-old cultures (three independent experiments with ten technical replicates for weight calculations and three independent experiments with two technical replicates for total stilbene measurement). Means followed by the same letter in one column were not different using one-way analysis of variance (ANOVA), followed by the Tukey HSD multiple comparison test.

Using HPLC, we determined the content and composition of stilbenes in the obtained $\mathrm{VaCML}$-transgenic cell lines. Overexpression of the VaCML65 gene led to a considerable increase in the content of stilbenes in all obtained transgenic lines in 3.8-23.7 times (Figure 2). Stilbene content in the VaCML65-transgenic cell lines reached $19.1 \mathrm{mg}$ per $\mathrm{g}$ of the dry cells weight (DW) and stilbene production reached $136 \mathrm{mg}$ per 1 (Figure 2, Table 1). This is one of the greatest values for cell cultures among the known data ([3], Figure 2, Table 1). This value was approximately 27 times higher than stilbene production in control cell culture KA0 (Table 1) and 4 times higher than stilbene production in VaCPK20 transgenic cell lines (up to $35 \mathrm{mg} / \mathrm{L},[19]$ ), but 1.2 times lower than that in the rolB transgenic cell culture of $V$. amurensis (152 $\mathrm{mg} / \mathrm{L}[16])$.

Considering other $\mathrm{VaCML}$ genes, we observed an increase in the content and production of stilbenes in the 52-2 and 93-1 cell lines transformed with the VaCML52 and VaCML93 genes (Figure 2; Table 1). We consider this effect rather as a non-specific transformation effect since only one cell line out of three for VaCML52 or VaCML93 showed significant changes in the production of stilbenes. All other cell lines transformed with VaCML52, VaCML86, VaCML93, and VaCML95 showed no significant changes in the content and production of stilbenes (Figure 2; Table 1). 


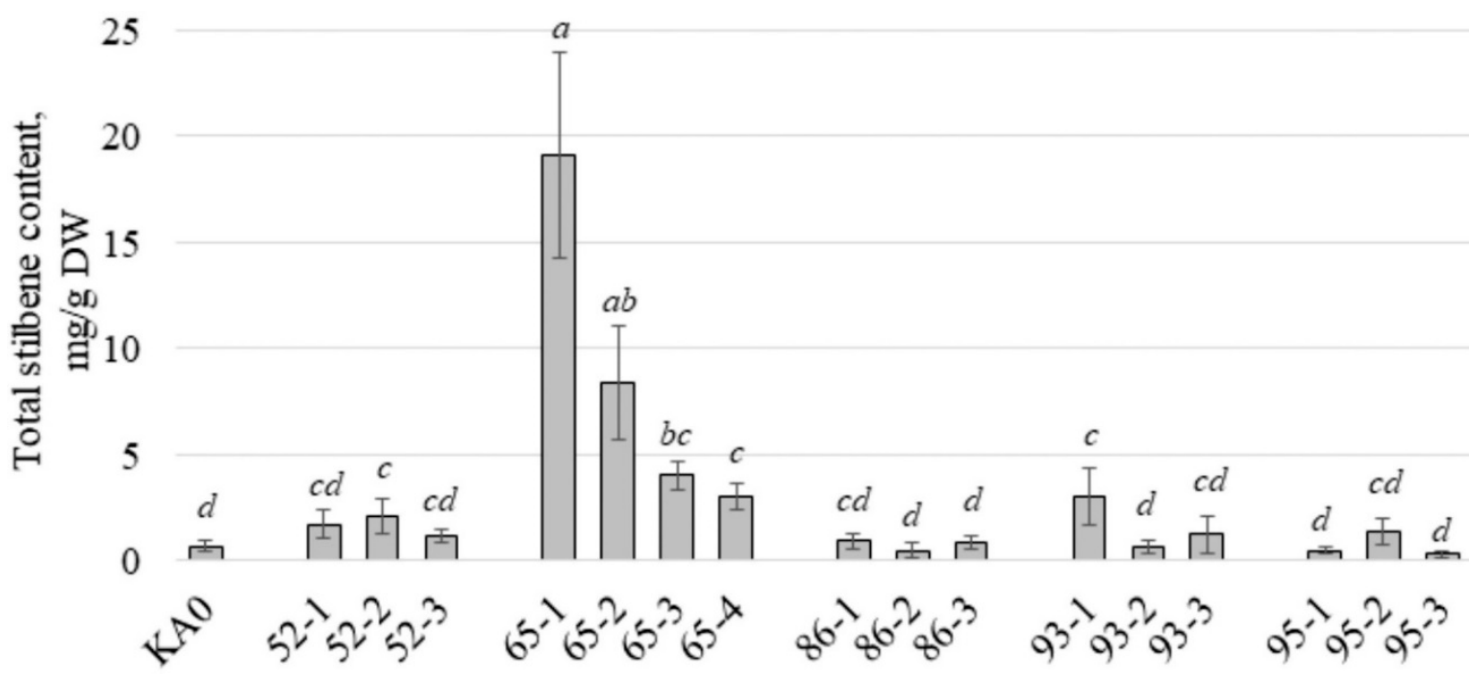

Figure 2. Total stilbene content in the cell lines of Vitis amurensis transformed with the VaCML52, VaCML65, VaCML86, VaCML93, or VaCML95 genes in mg per $\mathrm{g}$ of the dry weight (DW). KA0—control cell line transformed with the empty vector harboring only nptII selective marker; 52-1, 2, 3-cell lines transformed with the VaCML52 gene; 65-1, 2, 3, 4-cell lines transformed with the VaCML65 gene; 86-1, 2, 3-cell lines transformed with the VaCML86 gene; 93-1, 2, 3-cell lines transformed with the VaCML93 gene; 95-1, 2, 3-cell lines transformed with the VaCML95 gene. Means followed by the same letter were not different using one-way analysis of variance (ANOVA), followed by the Tukey HSD multiple comparison test (three independent experiments with two technical replicates). $p<0.05$ was considered statistically significant.

Overexpression of the $\mathrm{VaCML} 65$ gene did not change the spectrum of detected individual stilbenes (Table 2). The increase in the total content of stilbenes in the four VaCML65transgenic cell lines was due to a strong increase in the content of $t$-resveratrol (Table 2). For example, in the $\mathrm{VaCML65}$-transgenic lines, the content of $t$-resveratrol increased up to $18.1 \mathrm{mg} / \mathrm{g}$ DW, i.e., $4.6-43$ times in comparison with resveratrol levels detected in the control KA0 cell line (Table 2). The content of other stilbenoid compounds in the VaCML65transformed cells increased to a lesser extent or even decreased (Table 2). The data indicate that overexpression of the $V a C M L 65$ gene led to the increase in the content of stilbenes via activation of $t$-resveratrol biosynthesis (Table 2).

Then, it was important to verify whether the enhanced production of stilbenes in cell cultures overexpressing the VaCML65 gene was due to the activation of biosynthesis or to a reduction in the degradation of these compounds. For this purpose, we analyzed the expression of several important stilbene biosynthesis genes (Supplementary Figure S2), including five phenylalanine ammonia-lyase (PAL), ten stilbene synthase (STS), and five genes of VaMyb14, VaMyb15, VaMyb40, VaMyb60, and VaMyb107 transcription factors (Figure 3), which are known as possible positive regulators of stilbene biosynthesis [54]. Expression of these $V a M y b$ genes was considerably increased in grapevine cells with a high stilbene content [54].

We showed that overexpression of the $\mathrm{VaCML65}$ gene led to considerably increased mRNA transcript levels of the VaPAL1, 2, 5 (Figure 3a) and VaSTS2, 3, 4, 5, 6, 8, and 10 genes (Figure $3 \mathrm{~b}, \mathrm{c}$ ) in the four VaCML65-transgenic cell lines. The results show that the enhanced content of stilbenes in the obtained $\mathrm{VaCML65-transgenic} \mathrm{grape} \mathrm{cells} \mathrm{was} \mathrm{associated} \mathrm{with}$ activation of stilbene biosynthesis via a considerable increase in the expression of certain PAL and STS genes (Figure 3). Moreover, some Myb transcription factors may be involved in this process, for example, VaMyb60, because expression levels of VaMyb60 increased in all lines after transformation with $\mathrm{VaCML65}$ gene (Figure 3d). 

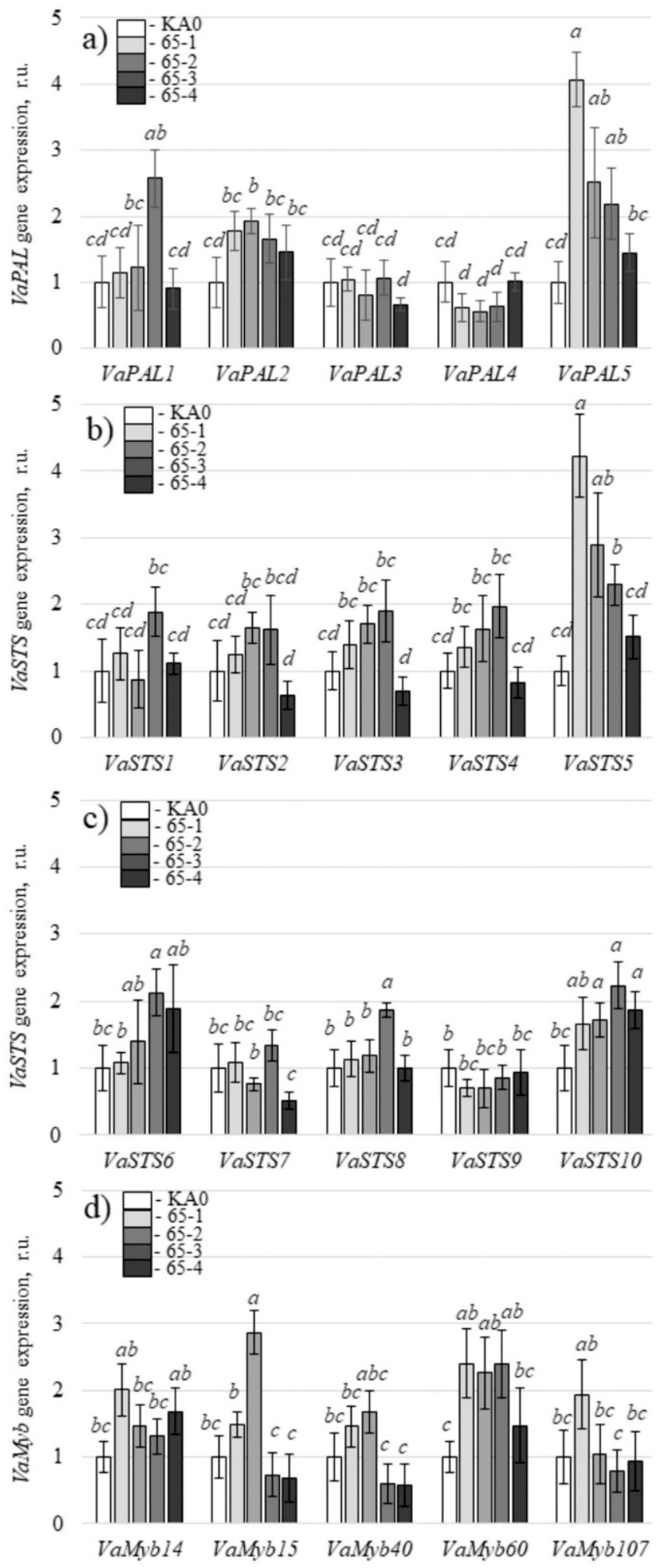

Figure 3. Quantification the VaPAL1-5 (a) and VaSTS1-5 (b), VaSTS6-10 (c), and VaMyb14, 15, 40, 60, and 107 (d) gene expression in the VaCML65-transgenic cell lines of Vitis amurensis performed by quantitative PCR (qRT-PCR). RNA was extracted from the vector control (KA0), VaCML65-1, $-2,-3$, and -4-transformed cell lines of $V$. amurensis. Means on each figure followed by the same letter were not different using one-way analysis of variance (ANOVA), followed by the Tukey HSD multiple comparison test (two independent experiments with eight technical replicates). $p<0.05$ was considered to be statistically significant. n.d.- - not detected. 
Table 2. The content of individual stilbenes (mg per g of the dry weight (DW)) in the transgenic Vitis amurensis cell lines transformed with VaCML52, VaCML65, VaCML86, VaCML93, or VaCML95 genes.

\begin{tabular}{|c|c|c|c|c|c|c|c|c|c|}
\hline Cell Lines & $\begin{array}{l}\text { Overexpressed } \\
C M L \text { Gene }\end{array}$ & $\begin{array}{c}t \text {-Resveratrol } \\
\text { Diglucoside }\end{array}$ & $t$-Piceid & $t$-Resveratrol & $\varepsilon$-Viniferin & $\delta$-Viniferin & cis-Resveratrol & cis-Piceid & Piceatannol \\
\hline KA0 & - & $0.12 \pm 0.07^{c}$ & $0.09 \pm 0.03^{b}$ & $0.42 \pm 0.11^{\mathrm{f}}$ & $0.05 \pm 0.02^{b}$ & $0.12 \pm 0.03^{b}$ & $0^{a}$ & $0^{a}$ & $0.01 \pm 0.01^{a}$ \\
\hline $52-1$ & \multirow{3}{*}{ VaCML52 } & $0.18 \pm 0.05^{c}$ & $0.08 \pm 0.03^{b}$ & $1.06 \pm 0.35$ ef & $0.08 \pm 0.04^{a b}$ & $0.28 \pm 0.09 \mathrm{ab}$ & $0.01 \pm 0.01^{a}$ & $0.01 \pm 0.01^{a}$ & $0.01 \pm 0.01^{a}$ \\
\hline $52-2$ & & $0.17 \pm 0.06^{c}$ & $0.07 \pm 0.03^{b}$ & $1.62 \pm 0.70 \mathrm{de}$ & $0.06 \pm 0.02^{b}$ & $0.17 \pm 0.05^{a b}$ & $0^{\mathrm{a}}$ & $0.01 \pm 0.01^{\mathrm{a}}$ & $0.01 \pm 0.01^{a}$ \\
\hline $52-3$ & & $0.23 \pm 0.09^{c}$ & $0.07 \pm 0.02^{b}$ & $0.58 \pm 0.21^{\mathrm{f}}$ & $0.09 \pm 0.03^{a b}$ & $0.18 \pm 0.06^{\mathrm{ab}}$ & $0^{a}$ & $0^{\mathrm{a}}$ & $0^{\mathrm{a}}$ \\
\hline $65-1$ & \multirow{4}{*}{ VaCML65 } & $0.26 \pm 0.09^{c}$ & $0.14 \pm 0.04^{b}$ & $18.06 \pm 7.09^{a}$ & $0.12 \pm 0.06^{\mathrm{ab}}$ & $0.46 \pm 0.16^{\mathrm{a}}$ & $0.01 \pm 0.01^{\mathrm{a}}$ & $0.01 \pm 0.01^{\mathrm{a}}$ & $0.02 \pm 0.01^{a}$ \\
\hline $65-2$ & & $0.25 \pm 0.08^{c}$ & $0.12 \pm 0.03^{b}$ & $7.79 \pm 3.31^{a b}$ & $0.06 \pm 0.03^{b}$ & $0.17 \pm 0.05^{a b}$ & $0^{\mathrm{a}}$ & $0.01 \pm 0.01^{\mathrm{a}}$ & $0.01 \pm 0.01^{a}$ \\
\hline $65-3$ & & $0.72 \pm 0.17^{\mathrm{a}}$ & $0.40 \pm 0.13^{a}$ & $2.31 \pm 0.47^{\mathrm{cd}}$ & $0.12 \pm 0.06^{a b}$ & $0.25 \pm 0.11^{a b}$ & $0^{a}$ & $0.01 \pm 0.01^{a}$ & $0.01 \pm 0.01^{a}$ \\
\hline $65-4$ & & $0.68 \pm 0.18^{a b}$ & $0.25 \pm 0.08^{a b}$ & $1.95 \pm 0.32^{\mathrm{cd}}$ & $0.08 \pm 0.02^{a b}$ & $0.35 \pm 0.14^{\mathrm{ab}}$ & $0^{\mathrm{a}}$ & $0.01 \pm 0.01^{a}$ & $0.01 \pm 0.01^{\mathrm{a}}$ \\
\hline $86-1$ & \multirow{3}{*}{ VaCML86 } & $0.22 \pm 0.11^{c}$ & $0.08 \pm 0.03^{b}$ & $0.33 \pm 0.12^{\mathrm{fg}}$ & $0.07 \pm 0.02^{b}$ & $0.25 \pm 0.09 \mathrm{ab}$ & $0.01 \pm 0.01^{\mathrm{a}}$ & $0^{\mathrm{a}}$ & $0.01 \pm 0.01^{a}$ \\
\hline $86-2$ & & $0.13 \pm 0.07^{c}$ & $0.05 \pm 0.03^{b}$ & $0.15 \pm 0.04 \mathrm{~g}$ & $0.08 \pm 0.05^{a b}$ & $0.19 \pm 0.11^{a b}$ & $0^{\mathrm{a}}$ & $0^{\mathrm{a}}$ & $0^{\mathrm{a}}$ \\
\hline $86-3$ & & $0.25 \pm 0.11^{c}$ & $0.08 \pm 0.03^{b}$ & $0.29 \pm 0.10^{\mathrm{fg}}$ & $0.05 \pm 0.02^{b}$ & $0.21 \pm 0.08^{a b}$ & $0.01 \pm 0.01^{a}$ & $0.01 \pm 0.01^{a}$ & $0.01 \pm 0.01^{a}$ \\
\hline $93-1$ & \multirow{3}{*}{$V a C M L 93$} & $0.14 \pm 0.05^{c}$ & $0.04 \pm 0.02^{b}$ & $2.32 \pm 1.03^{\mathrm{cd}}$ & $0.15 \pm 0.05^{a}$ & $0.37 \pm 0.11^{a b}$ & $0.01 \pm 0.01^{\mathrm{a}}$ & $0.01 \pm 0.01^{\mathrm{a}}$ & $0.01 \pm 0.01^{a}$ \\
\hline $93-2$ & & $0.31 \pm 0.12^{b c}$ & $0.07 \pm 0.03^{b}$ & $0.21 \pm 0.09 \mathrm{~g}$ & $0.02 \pm 0.01^{b}$ & $0.09 \pm 0.04^{b}$ & $0^{\mathrm{a}}$ & $0.02 \pm 0.01^{\mathrm{a}}$ & $0^{\mathrm{a}}$ \\
\hline $93-3$ & & $0.32 \pm 0.16^{\mathrm{bc}}$ & $0.11 \pm 0.06^{b}$ & $0.63 \pm 0.24^{\mathrm{f}}$ & $0.05 \pm 0.03^{b}$ & $0.17 \pm 0.10^{a b}$ & $0^{\mathrm{a}}$ & $0^{\mathrm{a}}$ & $0^{\mathrm{a}}$ \\
\hline $95-1$ & \multirow{3}{*}{ VaCML95 } & $0.16 \pm 0.06^{c}$ & $0.06 \pm 0.02^{b}$ & $0.15 \pm 0.06^{\mathrm{g}}$ & $0.03 \pm 0.01^{b}$ & $0.10 \pm 0.03^{b}$ & $0.01 \pm 0.01^{\mathrm{a}}$ & $0.01 \pm 0.01^{\mathrm{a}}$ & $0.01 \pm 0.01^{a}$ \\
\hline $95-2$ & & $0.23 \pm 0.07^{c}$ & $0.05 \pm 0.02^{b}$ & $1.01 \pm 0.37^{\text {ef }}$ & $0.03 \pm 0.02^{b}$ & $0.09 \pm 0.03^{b}$ & $0^{\mathrm{a}}$ & $0.01 \pm 0.01^{\mathrm{a}}$ & $0.01 \pm 0.01^{a}$ \\
\hline $95-3$ & & $0.11 \pm 0.03^{c}$ & $0.06 \pm 0.02^{b}$ & $0.14 \pm 0.07^{g}$ & $0.02 \pm 0.01^{b}$ & $0.08 \pm 0.03^{b}$ & $0^{a}$ & $0.01 \pm 0.01^{\mathrm{a}}$ & $0^{a}$ \\
\hline
\end{tabular}

KA0-control cell line of $V$. amurensis transformed with the "empty" vector harboring only nptII selective marker; 52-1, 2, 3-V. amurensis cell lines transformed with the VaCML52 gene; 65-1, 2, 3, 4-V. amurensis cell lines transformed with the VaCML65 gene; 86-1, 2, 3-V. amurensis cell lines transformed with the VaCML86 gene; 93-1, 2, 3-V. amurensis cell lines transformed with the VaCML93 gene; 95-1, 2, 3-V. amurensis cell lines transformed with the VaCML95 gene. The callus tissue samples were harvested from the 35-day-old cell cultures. Means followed by the same letter in one row were not different using one-way analysis of variance (ANOVA), followed by the Tukey HSD multiple comparison test (three independent experiments with two technical replicates). $p<0.05$ was considered statistically significant. 


\section{Conclusions}

In this paper, we investigated the effect of overexpression of five grapevine CML genes, which were highly up-regulated in wild-growing grapevine $V$. amurensis in response to stilbene-inducing conditions (stress hormones, phenolic precursors, and UV irradiation), and were suggested as promising candidates playing important roles in stilbene biosynthesis [43]. Previously, it was shown that up-regulation of the VaCML52, VaCML65, VaCML93, and $\mathrm{VaCML95}$ genes was induced after cultivation in the presence of SA, coumaric acid, MeJA, or UV treatment and associated with an increase in the content of stilbenes in both callus cell cultures and leaves of $V$. amurensis [43]. However, only VaCML65 overexpression considerably induced stilbene levels in all independent VaCML65-transgenic cell lines. The data indicate that the VaCML65 gene is involved in the signaling pathway regulating stilbene biosynthesis and acts as a positive regulator of $t$-resveratrol production. The proposed model of the signaling pathway leading to stilbene biosynthesis induction with the involvement of VaCML65 in this process was presented in Figure S2. Briefly, after signal perception, stimulated receptors are proposed to induce calcium influx, which then leads to the activation of calcium sensor proteins, including VaCML65. It is possible that VaCML65 together with mitogen-activated protein kinases (MAPK) cascade and hormone signaling could activate expression of $P A L, S T S$, and other genes responsible for stilbene biosynthesis, probably through activation of some transcription factors (e.g. VaMyb60).

The absence of a stable and considerable stilbene-modulating effect in the cell lines of $\mathrm{V}$. amurensis overexpressing the VaCML52, VaCML86, VaCML93, and VaCML95 genes indicates that these $\mathrm{VaCML}$ genes are not directly involved in the regulation of stilbene biosynthesis in grapevine. It is also possible that additional proteins, which are not present in the cell cultures, are necessary for the work of the VaCML52, VaCML86, VaCML93, and $\mathrm{VaCML95}$ for their implication as positive regulators for stilbene production. In conclusion, the results are important for understanding the signaling pathways and mechanisms regulating the biosynthesis of plant secondary metabolites and might be in demand in plant biotechnology and agriculture.

\section{Materials and Methods}

\subsection{Plant Material and Cell Cultures}

The V7 callus cultures were established in 2017 from young stems of the wild-growing mature $V$. amurensis vines near Vladivostok as described in Tyunin et al. 2019 [55]. All transgenic cell lines were obtained by Agrobacterium-mediated transformation as described in Aleynova-Shumakova et al., 2014 [19]. Briefly, the control KA0 cell culture transformed with the empty vector was obtained in 2020 by co-cultivation of the V7 cell suspension with Agrobacterium tumefaciens GV3101:pMP90 strain containing pZP-RCS2-nptII [56], which contained only the $\mathrm{Km}$ resistance gene, nptII.

To generate the construction for plant cell transformation, the full-length cDNA of VaCML52, VaCML65, VaCML86, VaCML93, and VaCML95 genes (GenBank accession number MN540595, MN540606, MN540576, MN540582, MN540584) were amplified by PCR using the primers presented in the Supplementary Table S1. The forward primer contained a BglII (for CML52), Psp124B I (or SacI, for CML65, 93, 95) or HindIII (for CML86) restriction site and the reverse primer contained a BamHI (for all CML genes) restriction site, which are underlined. The full-length cDNA of CMLs was cloned into the pSAT1 vector [56] by the BglII/Psp124B I/HindIII and BamHI sites under the control of the double cauliflower mosaic virus (CaMV 35S) promoter. Then, the expression cassette from pSAT1 with the CML genes was cloned into the pZP-RCS2-nptII vector [56] using the PalAI (AscI) sites. The pZP-RCS2-nptII construction also carried the nptII gene under the control of the double CaMV 35S promoter (Supplementary Figure S3). The used restriction enzymes were obtained from SibEnzyme (Novosibirsk, Russia).

The independently transformed $V a C M L$-transgenic callus cell lines of $V$. amurensis, designated 52-1, 52-2, 52-3 (VaCML52 gene); 65-1, 65-2, 65-3, 65-4 (VaCML65 gene); 86-1, 86-2, 86-3 (VaCML86 gene); 93-1, 93-2, 93-3 (VaCML93 gene); 95-1, 95-2, 95-3, 95-4 (VaCML95 
gene), were obtained in 2020 by transformation of the $\mathrm{V} 7$ cell suspension with $A$. tumefaciens strain GV3101::pMP90 containing pZP-RCS2-VaCML-nptII as described previously [19,57]. The $V a C M L$ genes and the selective marker nptII gene were driven under the double CaMV $35 \mathrm{~S}$ promoter. Transcript level of the nptII gene was verified using semiquantitative RT-PCR with the primers and PCR conditions described earlier [46]. The absence of A.tumefaciens was confirmed by RT-PCR of the VirB2 gene using primers listed in Table S1. All transgenic cell lines were cultivated on the solid Murashige and Skoog modified $\mathrm{W}_{\mathrm{B} / \mathrm{A}}$ medium [58] supplemented with $0.5 \mathrm{mg} / \mathrm{L} \mathrm{BAP,} 2 \mathrm{mg} / \mathrm{L} \mathrm{NAA}$, and $8 \mathrm{~g} / \mathrm{L}$ agar in the dark in $100 \mathrm{~mL}$ flasks with $20 \mathrm{~mL}$ of the medium. For stilbene analysis, the $V$. amurensis calli were cultivated at 35-day subculture intervals in the dark at $24-25^{\circ} \mathrm{C}$ in test tubes (height $150 \mathrm{~mm}$, internal diameter $146 \mathrm{~mm}$ ) with $7-8 \mathrm{~mL}$ of the $\mathrm{W}_{\mathrm{B} / \mathrm{A}}$ medium.

\subsection{Stilbene Analysis by High-Performance Liquid Chromatography (HPLC) and Mass Spectrometry}

The content of stilbenes was measured by HPLC as described [43,59]. Briefly, the extracts were separated on Zorbax C18 column (column temperature $40{ }^{\circ} \mathrm{C}, 150 \mathrm{~mm}$, 2.1-mm i.d., 3.5-lm part size, Agilent Technologies, Santa Clara, CA, USA) the on HPLC LC-20AD XR analytical system (Shimadzu, Kyoto, Japan). The mobile phase consisted of a gradient elution of $0.1 \%$ aqueous acetic acid (A) and acetonitrile (B). The gradient profile with a flow rate of $0.2 \mathrm{~mL} / \mathrm{min}$ was: $0 \mathrm{~min} 0 \% \mathrm{~B} ; 35 \mathrm{~min} 40 \% \mathrm{~B} ; 40 \mathrm{~min} 50 \% \mathrm{~B}$; $50 \mathrm{~min} 100 \% \mathrm{~B}$ and then eluent B until $60 \mathrm{~min}$. The injected volume was $5 \mu \mathrm{L}$. Liquid chromatography-high-resolution mass spectrometry for quantification of all components was performed using a 1260 Infinity analytical system (Agilent Technologies, USA) as described [20,59]. HPLC for quantification of all components was performed using LC-20 analytical HPLC system (Shimadzu, Japan) equipped with an SPD-M20A photodiode array detector, LC-20ADXR pump, Shim-pack XR-ODS II column and SIL-20ACXR auto sampler as described [46].

\subsection{RNA Isolation, Reverse Transcription and Quantitative Real-Time PCR (qRT-PCR)}

Total RNA extraction was performed using the cetyltrimethylammonium bromidebased extraction as described [47]. Complementary DNAs were synthesized using the MMLV Reverse transcription PCR Kit with oligo(dT)15 (RT-PCR, Evrogen, Moscow, Russia) as described [60]. qRT-PCRs were performed using the real-time PCR kit (Evrogen) and EvaGreen Real-time PCR dye (Biotium, Hayward, CA, USA) using total cDNAs as described $[28,46]$. The expression was calculated by the $2^{-\Delta \Delta C T}$ method with two internal controls, VaGAPDH and VaActin1, as described [61]. The primers used for qRT-PCRs are listed in Table S1. We used different primer sets for analyzing expression of the exogenous (transgene) and endogenous $\mathrm{VaCML}$ genes. To analyze transcript levels of the $\mathrm{VaCML}$ transgenes, the primers were designed according to the promoter and terminator sequences of the transgenes (Figure S4; Table S1). To analyze expression of the endogenous VaCML genes, the primers were designed to the untranslated (UTR) and specific coding gene regions of the VaCMLs (Figure S4; Table S1). To analyze transcript levels of both $V a C M L$ transgenes and $\mathrm{VaCML}$ endogenes (total expression), the primers were designed to protein-coding regions of $\mathrm{VaCML}$ genes (Figure S4; Table S1).

\subsection{Statistical Analysis}

For quantification of the $V a C M L, V a P A L$, VaSTS, and $V a M y b$ gene expression we used two independent experiments with eight technical replicates (four qPCR reactions normalized to $V a G A P D H$ and four qPCr reactions-to VaActin gene in each independent experiment). For callus tissue weight calculations, we used three independent experiments with ten technical replicates and three independent experiments with two technical replicates for total stilbene measurement. The data are presented as mean \pm standard error (SE) and were evaluated by one-way analysis of variance (ANOVA), followed by the Tukey HSD multiple comparison test performed in Excel using the XLSTAT software. A value of $p<0.05$ was considered significant. 
Supplementary Materials: The following supporting information can be downloaded at: https:// www.mdpi.com/article/10.3390/plants11020171/s1, Table S1: Primers used for amplification of Vitis amurensis cDNAs PCRs. Figure S1: Transcript levels of the nptII and VirB2 genes in grape cell cultures shown using separation of semiquantitative RT-PCR products of the $n p t I$ and VirB2 genes by gel electrophoresis. KA0 - control cell line of Vitis amurensis transformed with the "empty" vector harboring only nptII selective marker; 52-1, 2, 3-V. amurensis cell lines transformed with the VaCML52 gene; 65-1, 2, 3, 4-V. amurensis cell lines transformed with the VaCML65 gene; 86-1, 2, 3-V. amurensis cell lines transformed with the VaCML86 gene; 93-1, 2, 3-V. amurensis cell lines transformed with the VaCML93 gene; 95-1, 2, 3-V. amurensis cell lines transformed with the VaCML95 gene. Pc-positive control (pZP-RCS-nptII for nptII or Agrobacterium tumefaciens strain GV3101:pMP90 for VirB2); Ncnegative control (PCR mixture without DNA or bacteria). Figure S2: Proposed model of the signaling pathway leading to stilbene biosynthesis induction and VaCML65 functions in this process in the grapevine cells. Ultraviolet (UV), elicitors, pathogen effectors, and other environmental cues are perceived by specific receptors. Recognition of an external signal leads to sustained calcium influx, activation of VaCML65 and other calcium sensors, MAPK cascades, which promotes plant hormone signaling and TF activation. TFs activate transcription of the genes responsible for stilbene biosynthesis. MAPK—-mitogen activated protein kinases; CMLs—calmodulin-like proteins; TF-transcription factors. Figure S3: Schematic representation of the pZP-RCS2-nptII-VaCML vector [46] carrying the $\mathrm{VaCML}$ genes. $\mathrm{RB}$ and $\mathrm{LB}$ - correspond to the sequences of the right and left borders of T-DNA; 2*35S - the double 35S promoter of the cauliflower mosaic virus (CaMV); ter-CaMV 35S terminator; $n p t \mathrm{II}$-kanamycin resistance gene; $\mathrm{Sp}$-spectinomycin resistance gene. Figure S4: Schematic representation of the T-DNA region from the pZP-RCS2-nptII-VaCML vector (a) and the endogenous $\mathrm{VaCML}$ gene of Vitis amurensis (b) with the used primer sets for analyzing the transgene $\mathrm{VaCML}$ and endogenous $\mathrm{VaCML}$ transcript levels. 2*35S - the double $35 \mathrm{~S}$ promoter of the cauliflower mosaic virus (CaMV); ter-CaMV 35S terminator; VaCML—the coding sequence of the VaCML52, VaCML65, VaCML86, VaCML93, or VaCML95 genes; 5'UTR and 3'UTR - untranslated regions of the VaCML genes. CML-S and pSAT-term-A-primers for qRT-PCR estimation of the transgene VaCML52, 65, 86, and 93 expression; pSAT-prom-S and CML95-A - primers for qRT-PCR estimation of the transgene VaCML95 expression; CML-S and CML-endo-A-primers for qRT-PCR estimation of the endogenous VaCML52, 65, 86, and 93 expression; CML-endo-S and CML95-A-primers for qRT-PCR estimation of the endogenous $\mathrm{VaCML95}$ expression; CML-S and CML-A - primers for qRT-PCR estimation of the total VaCML52, 65, 86, and 93 expression; CML95-S and CML95-A-primers for qRT-PCR estimation of the total $\mathrm{VaCML95}$ expression.

Author Contributions: A.S.D. and K.V.K. performed research design, interpretation and paper preparation. O.A.A. and A.R.S. performed RNA isolations, experiments with cell cultures, data analysis. A.R.S. performed HPLC analysis. Z.V.O., A.A.A. and N.N.N. performed qRT-PCRs and participated in data analysis. All authors have read and agreed to the published version of the manuscript.

Funding: This work was supported by the grant 19-04-00063 from the Russian Foundation for Basic Research.

Institutional Review Board Statement: Not applicable.

Informed Consent Statement: Not applicable.

Data Availability Statement: The data presented in this study are available in all Tables and Figures of the manuscript.

Conflicts of Interest: The authors declare no conflict of interest.

\section{References}

1. Langcake, P.; Pryce, R.J. A new class of phytoalexins from grapevines. Experientia 1977, 33, 151-152. [CrossRef] [PubMed]

2. Kiselev, K.V. Perspectives for production and application of resveratrol. Appl. Microbiol. Biotechnol. 2011, 90, 417-425. [CrossRef]

3. Dubrovina, A.S.; Kiselev, K.V. Regulation of stilbene biosynthesis in plants. Planta 2017, 346, 597-623. [CrossRef] [PubMed]

4. Colica, C.; Milanovic, M.; Milic, N.; Aiello, V.; De Lorenzo, A.; Abenavoli, L. A systematic review on natural antioxidant properties of resveratrol. Nat. Prod. Commun. 2018, 13, 1195-1203. [CrossRef]

5. Shaito, A.; Posadino, A.M.; Younes, N.; Hasan, H.; Halabi, S.; Alhababi, D.; Al-Mohannadi, A.; Abdel-Rahman, W.M.; Eid, A.H.; Nasrallah, G.K.; et al. Potential adverse effects of resveratrol: A literature review. Int. J. Mol. Sci. 2020, 21, 2084. [CrossRef] 
6. $\quad$ Sikuten, I.; Stambuk, P.; Andabaka, Z.; Tomaz, I.; Markovic, Z.; Stupic, D.; Maletic, E.; Kontic, J.K.; Preiner, D. Grapevine as a rich source of polyphenolic compounds. Molecules 2020, 25, 5604. [CrossRef]

7. Jeandet, P.; Delaunois, B.; Conreux, A.; Donnez, D.; Nuzzo, V.; Cordelier, S.; Clément, C.; Courot, E. Biosynthesis, metabolism, molecular engineering, and biological functions of stilbene phytoalexins in plants. Biofactors 2010, 36, 331-341. [CrossRef] [PubMed]

8. Riccio, B.V.F.; Fonseca-Santos, B.; Ferrari, P.C.; Chorilli, M. Characteristics, biological properties and analytical methods of trans-resveratrol: A review. Crit. Rev. Anal. Chem. 2020, 50, 339-358. [CrossRef]

9. Chong, J.L.; Poutaraud, A.; Hugueney, P. Metabolism and roles of stilbenes in plants. Plant Sci. 2009, 177, 143-155. [CrossRef]

10. Faurie, B.; Cluzet, S.; Mérillon, J.M. Implication of signaling pathways involving calcium, phosphorylation and active oxygen species in methyl jasmonate-induced defense responses in grapevine cell cultures. J. Plant Physiol. 2009, 166, 1863-1877. [CrossRef]

11. Vandelle, E.; Vannozzi, A.; Wong, D.; Danzi, D.; Digby, A.M.; Dal Santo, S.; Astegno, A. Identification, characterization, and expression analysis of calmodulin and calmodulin-like genes in grapevine (Vitis vinifera) reveal likely roles in stress responses. Plant Physiol. Biochem. 2018, 129, 221-237. [CrossRef]

12. Belchi-Navarro, S.; Almagro, L.; Sabater-Jara, A.B.; Fernandez-Perez, F.; Bru, R.; Pedreno, M.A. Induction of trans-resveratrol and extracellular pathogenesis-related proteins in elicited suspension cultured cells of Vitis vinifera cv Monastrell. J. Plant Physiol. 2013, 170, 258-264. [CrossRef]

13. Nicolas, P.; Lecourieux, D.; Kappel, C.; Cluzet, S.; Cramer, G.; Delrot, S.; Lecourieux, F. The basic leucine zipper transcription factor ABSCISIC ACID RESPONSE ELEMENT-BINDING FACTOR2 is an important transcriptional regulator of abscisic acid-dependent grape berry ripening processes. Plant Physiol. 2014, 164, 365-383. [CrossRef]

14. Xu, A.; Zhan, J.C.; Huang, W.D. Effects of ultraviolet C, methyl jasmonate and salicylic acid, alone or in combination, on stilbene biosynthesis in cell suspension cultures of Vitis vinifera L. cv. Cabernet Sauvignon. Plant Cell Tissue Organ Cult. 2015, 122, 197-211. [CrossRef]

15. Degu, A.; Ayenew, B.; Cramer, G.R.; Fait, A. Polyphenolic responses of grapevine berries to light, temperature, oxidative stress, abscisic acid and jasmonic acid show specific developmental-dependent degrees of metabolic resilience to perturbation. Food Chem. 2016, 212, 828-836. [CrossRef] [PubMed]

16. Dubrovina, A.S.; Kiselev, K.V.; Veselova, M.V.; Isaeva, G.A.; Fedoreyev, S.A.; Zhuravlev, Y.N. Enhanced resveratrol accumulation in rolB transgenic cultures of Vitis amurensis correlates with unusual changes in CDPK gene expression. J. Plant Physiol. 2009, 166, 1194-1206. [CrossRef] [PubMed]

17. Kiselev, K.V.; Shumakova, O.A.; Manyakhin, A.Y.; Mazeika, A.N. Influence of calcium influx induced by the calcium ionophore, A23187, on resveratrol content and the expression of CDPK and STS genes in the cell cultures of Vitis amurensis. Plant Growth Regul. 2012, 68, 371-381. [CrossRef]

18. Kiselev, K.V.; Shumakova, O.A.; Manyakhin, A.Y. Effects of the calmodulin antagonist W7 on resveratrol biosynthesis in Vitis amurensis Rupr. Plant Mol. Biol. Rep. 2013, 31, 1569-1575. [CrossRef]

19. Aleynova-Shumakova, O.A.; Dubrovina, A.S.; Manyakhin, A.Y.; Karetin, Y.A.; Kiselev, K.V. VaCPK20 gene overexpression significantly increased resveratrol content and expression of stilbene synthase genes in cell cultures of Vitis amurensis Rupr. Appl. Microbiol. Biotechnol. 2014, 98, 5541-5549. [CrossRef]

20. Aleynova, O.A.; Dubrovina, A.S.; Manyakhin, A.Y.; Karetin, Y.A.; Kiselev, K.V. Regulation of resveratrol production in Vitis amurensis cell cultures by calcium-dependent protein kinases. Appl. Biochem. Biotechnol. 2015, 175, 1460-1476. [CrossRef]

21. Hoell, J.; Vannozzi, A.; Czemmel, S.; D’Onofrio, C.; Walker, A.R.; Rausch, T.; Lucchin, M.; Boss, P.K.; Dry, I.B.; Bogs, J. The R2R3-MYB transcription factors MYB14 and MYB15 regulate stilbene biosynthesis in Vitis vinifera. Plant Cell 2013, 25, 4135-4149. [CrossRef]

22. Fang, L.; Hou, Y.; Wang, L.; Xin, H.; Wang, N.; Li, S. Myb14, a direct activator of STS, is associated with resveratrol content variation in berry skin in two grape cultivars. Plant Cell Rep. 2014, 33, 1629-1640. [CrossRef]

23. Kiselev, K.V.; Aleynova, O.A.; Ogneva, Z.V.; Suprun, A.R.; Ananev, A.A.; Nityagovsky, N.N.; Dubrovina, A.S. The effect of stress hormones, UV-C, and stilbene precursors on calmodulin $(\mathrm{C} a \mathrm{M})$ and calmodulin-like gene $(C M L)$ expression in Vitis amurensis Rupr. Plant Cell Tissue Organ Cult. 2021, 146, 59-68. [CrossRef]

24. Choi, W.G.; Hilleary, R.; Swanson, S.J.; Kim, S.H.; Gilroy, S. Rapid, long-distance electrical and calcium signaling in plants. Annu. Rev. Plant Biol. 2016, 67, 287-307. [CrossRef] [PubMed]

25. Kudla, J.; Becker, D.; Grill, E.; Hedrich, R.; Hippler, M.; Kummer, U.; Parniske, M.; Romeis, T.; Schumacher, K. Advances and current challenges in calcium signaling. New Phytol. 2018, 218, 414-431. [CrossRef] [PubMed]

26. DeFalco, T.A.; Bender, K.W.; Snedden, W.A. Breaking the code: $\mathrm{Ca}^{2+}$ sensors in plant signaling. Biochem. J. 2010, 425, 27-40. [CrossRef]

27. Mohanta, T.K.; Yadav, D.; Khan, A.L.; Hashem, A.; Abd Allah, E.F.; Al-Harrasi, A. Molecular players of EF-hand containing calcium signaling event in plants. Int. J. Mol. Sci. 2019, 20, 1476. [CrossRef]

28. McCormack, E.; Braam, J. Calmodulins and related potential calcium sensors of Arabidopsis. New Phytol. 2003, 159, 585-598 [CrossRef]

29. Dubrovina, A.S.; Aleynova, O.A.; Ogneva, Z.V.; Suprun, A.R.; Ananev, A.A.; Kiselev, K.V. The effect of abiotic stress conditions on expression of calmodulin ( $\mathrm{CaM})$ and calmodulin-like (CML) genes in wild-growing grapevine Vitis amurensis. Plants 2019, 8, 602. [CrossRef] 
30. Batistič, O.; Kudla, J. Analysis of calcium signaling pathways in plants. Biochim. Biophys. Acta 2012, 1820, 1283-1293. [CrossRef]

31. Dobney, S.; Chiasson, D.; Lam, P.; Smith, S.P.; Snedden, W.A. The calmodulin-related calcium sensor CML42 plays a role in trichome branching. J. Biol. Chem. 2009, 284, 31647-31657. [CrossRef]

32. Cheval, C.; Aldon, D.; Galaud, J.P.; Ranty, B. Calcium/calmodulin-mediated regulation of plant immunity. Biochim. Biophys. Acta 2013, 1833, 1766-1771. [CrossRef]

33. Zeng, H.; Xu, L.; Singh, A.; Wang, H.; Du, L.; Poovaiah, B.W. Involvement of calmodulin and calmodulin-like proteins in plant responses to abiotic stresses. Front. Plant Sci. 2015, 6, 600. [CrossRef]

34. Midhat, U.; Ting, M.K.Y.; Teresinski, H.J.; Snedden, W.A. The calmodulin-like protein, CML39, is involved in regulating seed development, germination, and fruit development in Arabidopsis. Plant Mol. Biol. 2018, 96, 375-392. [CrossRef] [PubMed]

35. Shen, L.; Yang, S.; Guan, D.Y.; He, S.L. CaCML13 acts positively in pepper immunity against Ralstonia solanacearum infection forming feedback loop with CabZIP63. Int. J. Mol. Sci. 2020, 21, 4186. [CrossRef] [PubMed]

36. Vanderbeld, B.; Snedden, W.A. Developmental and stimulus-induced expression patterns of Arabidopsis calmodulin-like genes CML37, CML38 and CML39. Plant Mol. Biol. 2007, 64, 683-697. [CrossRef] [PubMed]

37. Bender, K.W.; Dobney, S.; Ogunrinde, A.; Chiasson, D.; Mullen, R.T.; Teresinski, H.J.; Singh, P.; Munro, K.; Smith, S.P.; Snedden, W.A. The calmodulin-like protein CML43 functions as a salicylic-acid-inducible root-specific $\mathrm{Ca}^{2+}$ sensor in Arabidopsis. Biochem. J. 2014, 457, 127-136. [CrossRef]

38. Lu, L.; Rong, W.; Zhou, R.; Huo, N.; Zhang, Z. TaCML36, a wheat calmodulin-like protein, positively participates in an immune response to Rhizoctonia cerealis. Crop J. 2019, 7, 608-618. [CrossRef]

39. Wan, D.L.; Li, R.L.; Zou, B.; Zhang, X.; Cong, J.Y.; Wang, R.G.; Xia, Y.J.; Li, G.J. Calmodulin-binding protein CBP60g is a positive regulator of both disease resistance and drought tolerance in Arabidopsis. Plant Cell Rep. 2012, 31, 1269-1281. [CrossRef]

40. Rao, S.S.; El-Habbak, M.H.; Havens, W.M.; Singh, A.; Zheng, D.M.; Vaughn, L.; Haudenshield, J.S.; Hartman, G.L.; Korban, S.S.; Ghabrial, S.A. Overexpression of GmCaM4 in soybean enhances resistance to pathogens and tolerance to salt stress. Mol. Plant Pathol. 2014, 15, 145-160. [CrossRef]

41. Munir, S.; Liu, H.; Xing, Y.; Hussain, S.; Ouyang, B.; Zhang, Y.; Li, H.; Ye, Z. Overexpression of calmodulin-like (ShCML44) stress-responsive gene from Solanum habrochaites enhances tolerance to multiple abiotic stresses. Sci. Rep. 2016, 6, 31772. [CrossRef]

42. Kalaipandian, S.; Xue, G.P.; Rae, A.L.; Glassop, D.; Bonnett, G.D.; McIntyre, L.C. Overexpression of TaCML20, a calmodulin-like gene, enhances water soluble carbohydrate accumulation and yield in wheat. Physiol. Plant. 2019, 165, 790-799. [CrossRef]

43. Ma, Q.P.; Zhou, Q.Q.; Chen, C.M.; Cui, Q.Y.; Zhao, Y.X.; Wang, K.; Arkorful, E.; Chen, X.; Sun, K.; Li, X.H. Isolation and expression analysis of CsCML genes in response to abiotic stresses in the tea plant (Camellia sinensis). Sci. Rep. 2019, 9, 8211. [CrossRef]

44. Ma, Y.Y.; Zhang, Y.L.; Shao, H.; Lu, J. Differential physio-biochemical responses to cold stress of cold-tolerant and non-tolerant grapes (Vitis L.) from China. J. Agron. Crop Sci. 2010, 196, 212-219. [CrossRef]

45. Liu, L.; Li, H. Review research progress in amur grape, Vitis amurensis Rupr. Can. J. Plant Sci. 2013, 93, 565-575. [CrossRef]

46. Kiselev, K.V.; Aleynova, O.A.; Grigorchuk, V.P.; Dubrovina, A.S. Stilbene accumulation and expression of stilbene biosynthesis pathway genes in wild grapevine Vitis amurensis Rupr. Planta 2017, 245, 151-159. [CrossRef]

47. Eamens, A.; Wang, M.B.; Smith, N.A.; Waterhouse, P.M. RNA silencing in plants: Yesterday, today, and tomorrow. Plant Physiol. 2008, 147, 456-468. [CrossRef] [PubMed]

48. Velten, J.; Cakir, C.; Youn, E.; Chen, J.; Cazzonelli, C.I. Transgene silencing and transgene-derived siRNA production in tobacco plants homozygous for an introduced AtMYB90 construct. PLoS ONE 2012, 7, e30141. [CrossRef]

49. Wilson, R.C.; Doudna, J.A. Molecular mechanisms of RNA interference. Annu. Rev. Biophys. 2013, 42, 217-239. [CrossRef] [PubMed]

50. Rosa, C.; Kuo, Y.W.; Wuriyanghan, H.; Falk, B.W. RNA interference mechanisms and applications in plant pathology. Annu. Rev. Phytopathol. 2018, 56, 581-610. [CrossRef]

51. Dadami, E.; Moser, M.; Zwiebel, M.; Krczal, G.; Wassenegger, M.; Dalakouras, A. An endogene-resembling transgene delays the onset of silencing and limits siRNA accumulation. FEBS Lett. 2013, 18, 706-710. [CrossRef]

52. Dadami, E.; Dalakouras, A.; Zwiebel, M.; Krczal, G.; Wassenegger, M. An endogene-resembling transgene is resistant to DNA methylation and systemic silencing. RNA Biol. 2014, 11, 934-941. [CrossRef] [PubMed]

53. Suprun, A.R.; Ogneva, Z.V.; Dubrovina, A.S.; Kiselev, K.V. Effect of spruce PjSTS1a, PjSTS2, or PjSTS3 gene overexpression on stilbene biosynthesis in callus cultures of Vitis amurensis Rupr. Biotechnol. Appl. Biochem. 2020, 67, 234-239. [CrossRef] [PubMed]

54. Kiselev, K.V.; Aleynova, O.A.; Tyunin, A.P. Expression of the R2R3 MYB transcription factors in Vitis amurensis Rupr. plants and cell cultures with different resveratrol content. Russ. J. Genet. 2017, 53, 465-471. [CrossRef]

55. Tyunin, A.P.; Suprun, A.R.; Nityagovsky, N.N.; Manyakhin, A.Y.; Karetin, Y.A.; Dubrovina, A.S.; Kiselev, K.V. The effect of explant origin and collection season on stilbene biosynthesis in cell cultures of Vitis amurensis Rupr. Plant Cell Tissue Organ Cult. 2019, 136, 189-196. [CrossRef]

56. Tzfira, T.; Tian, G.W.; Lacroix, B.; Vyas, S.; Li, J.; Leitner-Dagan, Y.; Krichevsky, A.; Taylor, T.; Vainstein, A.; Citovsky, V. pSAT vectors: A modular series of plasmids for autofluorescent protein tagging and expression of multiple genes in plants. Plant Mol. Biol. 2005, 57, 503-516. [CrossRef]

57. Kiselev, K.V.; Dubrovina, A.S.; Shumakova, O.A.; Karetin, Y.A.; Manyakhin, A.Y. Structure and expression profiling of a novel calcium-dependent protein kinase gene, $C D P K 3 a$, in leaves, stems, grapes, and cell cultures of wild-growing grapevine Vitis amurensis Rupr. Plant Cell Rep. 2013, 32, 431-442. [CrossRef] 
58. Dubrovina, A.S.; Manyakhin, A.Y.; Zhuravlev, Y.N.; Kiselev, K.V. Resveratrol content and expression of phenylalanine ammonialyase and stilbene synthase genes in rolC transgenic cell cultures of Vitis amurensis. Appl. Microbiol. Biotechnol. 2010, 88, 727-736. [CrossRef]

59. Aleynova, O.A.; Grigorchuk, V.P.; Dubrovina, A.S.; Rybin, V.G.; Kiselev, K.V. Stilbene accumulation in cell cultures of Vitis amurensis Rupr. overexpressing VaSTS1, VaSTS2, and VaSTS7 genes. Plant Cell Tissue Organ Cult. 2016, 125, 329-339. [CrossRef]

60. Kiselev, K.V.; Ogneva, Z.V.; Suprun, A.R.; Grigorchuk, V.P.; Dubrovina, A.S. Action of ultraviolet-C radiation and p-coumaric acid on stilbene accumulation and expression of stilbene biosynthesis-related genes in the grapevine Vitis amurensis Rupr. Acta Physiol. Plant 2019, 41, 28. [CrossRef]

61. Livak, K.J.; Schmittgen, T.D. Analysis of relative gene expression data using real-time quantitative PCR and the $2^{-\Delta \Delta C T}$ method. Methods 2001, 25, 402-408. [CrossRef] [PubMed] 